\title{
Un abordaje del concepto 'seguridad energética' a la luz de los desarrollos del género historia conceptual
}

\author{
María Noel Dussort*
}

\section{Resumen:}

Cada concepto tiene una historia. Precisamente, el género historia conceptual permite reconstruir aquellos momentos de crisis en donde el vocabulario que dotaba de sentido al mundo pierde vigencia y es reformateado por las transformaciones del contexto histórico. En este sentido cabe preguntarse con Reinhart Koselleck, uno de los exponentes más importantes de este género, ¿cuál es la naturaleza de la relación temporal entre conceptos y circunstancias?

En nuestros días, el concepto 'seguridad energética' ha derivado en múltiples significados, muchas veces de carácter determinista, dando a conocer que no hay consenso sobre una sola definición. En consecuencia, el objetivo de este trabajo es analizar el concepto de seguridad energética a partir de los desarrollos del género historia conceptual con el fin de examinar, por un lado, su evolución a la luz de los acontecimientos a nivel internacional que provocaron su surgimiento y su modificación y, por otro lado, dilucidar si dicho concepto apareció ligado a la planificación de las políticas públicas (política energética), o por el contrario, si fueron las políticas públicas las que derivaron de aquel significante. En definitiva, se intenta desandar la constitución de esta noción desde la disciplina de las Relaciones Internacionales.

Palabras claves: seguridad energética - historia conceptual

An approach of the energy security concept under conceptual history studies

\begin{abstract}
:
Each concept has a history. Conceptual history studies allow for rebuilding those moments of crisis where vocabulary which used to make sense to the world, becomes outdate. Therefore such vocabulary has to be updated regarding the transformations within the historical context. In this sense Reinhart Koselleck, one of the main exponents of conceptual history studies, asks about what is the nature of the time relationship between concepts and circumstances. Now at day 'energy security' as a concept has many meanings, most of them are deterministic ones, showing that there is no consensus about one single definition of energy security. Consequently, the aim of this article is to analyze the concept of energy security under the conceptual history approach to study its evolution regarding the changes at the international system level which caused the appearance of the concept itself and its modifications. Apart from that, this article could help to explain if the concept of energy security appeared bound to the public politics (energy policy) or there were the public politics which came from energy security. We try to go back along the constitution of the energy security notion since the International Relations discipline.
\end{abstract}

Key words: energy security - conceptual history

Trabajo enviado: 19/03/2017 Trabajo aceptado: $31 / 05 / 2017$

\footnotetext{
* Licenciada en Relaciones Internacionales y estudiante del Doctorado en Relaciones Internacionales de la Facultad de Ciencia Política y Relaciones Internacionales de la Universidad Nacional de Rosario (UNR). Becaria doctoral del Consejo Nacional de Investigaciones Científicas y Técnicas (CONICET), Instituto de Investigaciones de la Facultad de Ciencia Política y Relaciones Internacionales de la UNR. Docente de la Facultad de Ciencia Política y Relaciones Internacionales de la UNR y Coordinadora del Grupo de Estudios sobre India de Rosario (GEIR), Programa de Estudios sobre Relaciones y Cooperación Sur-Sur (PRECSUR) de la Facultad de Ciencia Política y Relaciones Internacionales de la UNR. Correo electrónico: maria.dussort@fcpolit.unr.edu.ar
} 
Cada concepto tiene una historia. "Precisamente porque cada palabra puede tener una multiplicidad de significados que se van adecuando a la realidad mudable (...)" (KOSELLECK, 2004: 30). A partir de esta afirmación, nos interesa realizar un ejercicio de aplicación del género historia conceptual [Begriffsgeschichte] a un concepto tratado por las Relaciones Internacionales, es decir, la seguridad energética. En especial porque en nuestros días dicho concepto ha derivado en múltiples significados, muchas veces de carácter determinista, dando a conocer que no hay consenso en la academia sobre una sola definición.

Siguiendo esta idea, Reinhart Koselleck (2004: 35) entiende al concepto histórico fundamental como aquel que "(...) en combinación con varias docenas de otros conceptos de similar importancia, dirige e informa por entero el contenido político y social de una lengua". Entonces, de la misma manera que el autor distingue las distintas variantes en las que derivó y se combinó el concepto de Estado (en Estado monárquico, Estado republicano, etc.), nosotros pretendemos demostrar que el concepto histórico fundamental de seguridad se ha amalgamado con otros, adjetivando al sujeto y dando aparición a unidades conceptuales como seguridad alimentaria, seguridad ambiental, seguridad humana o el término al que nos dedicaremos: seguridad energética.

Antes de adentrarnos en la explicación que nos atañe, es importante aclarar la naturaleza del análisis utilizado. "La historia conceptual es un género desarrollado desde mediados del siglo XX en Alemania que en su momento quiso diferenciarse de otras formas de trabajar con los conceptos del mundo alemán. Especialmente de aquellos que separaban ideas y conceptos de sus contextos de producción" (LESGART, 2011: 3). Tal es así que la Begriffsgeschichte no trata el lenguaje político como un discurso autónomo, ni como un producto de la ideología, de la estructura social o de la manipulación de la elite. Muy por el contrario, el campo de la acción es definido por el lenguaje. En este sentido, historiadores conceptuales como Koselleck, Gadamer o Richter sitúan la formación de un concepto y su uso en los contextos históricos.

En consecuencia, el objetivo de este trabajo es realizar un análisis histórico conceptual de la seguridad energética a partir de los desarrollos epistemológicos de Reinhart Koselleck, con el fin de, por un lado, examinar su evolución a la luz de los acontecimientos a nivel internacional que provocaron su surgimiento y su modificación y, por el otro, dilucidar si dicho concepto apareció ligado a la planificación de las políticas públicas (política energética), o si fueron las políticas públicas las que derivaron de aquel significante

Debemos partir de la consideración que en la actualidad "seguridad energética" es admitida por la comunidad académica como una unidad conceptual, ya que parte de la unión de dos términos diferentes. Por esta razón, el presente trabajo conllevará el análisis de la historia conceptual de los dos significantes que hacen al concepto, a saber seguridad y energía y como confluyeron.

Desde la lógica lingüística Koselleck, retomando a Heiner Schultz, mostró que existen:

cuatro posibilidades para analizar el cambio recíproco de los conceptos y las circunstancias. [Primero], el significado de la palabra, así como el de las circunstancias aprehendidas en ella permanecen sincrónica y diacrónicamente constantes. [Segundo], el significado de la palabra permanece constante, pero las circunstancias cambian, distanciándose del antiguo significado. La realidad así transformada debe ser nuevamente conceptualizada. [Tercero], el significado de la palabra cambia, pero la realidad previamente aprehendida por ella permanece constante. Por tanto, la semántica debe encontrar una nueva forma de expresión con el fin de ajustarse de nuevo fielmente a esa realidad. [Cuarto], las circunstancias y el significado de las palabras se desarrollan separadamente, de manera que la correspondencia inicial no puede mantenerse por más tiempo (KOSELLECK, 2004: 31).

Tomando las anteriores consideraciones, se intentará identificar en cuáles de estas posibilidades de análisis entre conceptos y circunstancias se puede incluir la historia del 
concepto de seguridad energética. Al respecto, según Lesgart (2001: 4) la combinación entre la diacronía y la sincronía realizado por Koselleck "permite identificar con mayor eficacia la permanencia de experiencias anteriores; las continuidades; las innovaciones y alteraciones en el uso (...)".

Entonces, “¿cuál es la naturaleza de la relación temporal entre conceptos y situaciones o circunstancias? Sin duda, aquí está la clave para la historia de los conceptos, porque cualquier cosa que pueda y deba ser conceptualizada se encuentra fuera de los conceptos" (KOSELLECK, 2004: 30). Al amparo de esta motivación del autor, en los párrafos siguientes indagaremos en las "situaciones o circunstancias" que llevaron a la mutación del concepto elegido.

El trabajo comienza con el abordaje del concepto de seguridad energética desde la seguridad nacional. A continuación se analizan las mutaciones que sufrió a consecuencia del escenario económico cambiante de los años 70'. Luego, se hace referencia a la forma en que comenzó a ser considerado como un concepto multidimensional en el orden internacional de Posguerra Fría. Finalmente, se analizan los atentados del 11 de septiembre de 2001 en Estados Unidos como un punto de ruptura a nivel internacional que tuvo sus repercusiones en el concepto aquí estudiado y, a partir de entonces, la forma en que ha evolucionado hasta el tiempo presente.

\section{Seguridad energética. El abordaje desde la seguridad nacional}

Según Lesgart (2001:4), "la historia conceptual es especialmente útil para reconstruir aquellos momentos de crisis (...) en donde el vocabulario que dotaba de sentido al mundo se desvanece, o entra en discrepancia con nuevos términos". Tal es así que justamente son los momentos de cambios rotundos en el sistema internacional que van a llevar a ilustrar esta afirmación.

Partimos del hecho puntual que la unidad conceptual seguridad energética proviene del concepto de seguridad nacional, asociado a la noción de protección y a la supervivencia física del Estado-nación. Su acercamiento cognoscitivo, desde el área de las Relaciones Internacionales comenzó durante el período entre guerras y se afianzó en el contexto internacional de Guerra Fría.

Una de las circunstancias clave, que posteriormente fue reconocida por los académicos como punto de inicio de la construcción del concepto de seguridad energética, se produjo al aproximarse la Primera Guerra Mundial. Con el inicio del siglo XX, se promovieron una serie de cambios técnicos en el sector armamentístico que repercutieron en la política estatal de la época, con implicancias contundentes en la estrategia de abastecimiento energético soberano.

Un acontecimiento que rompió con la regularidad establecida en el sector militar se originó en 1912 cuando el Primer Lord del Almirantazgo británico, Winston Churchill, decidió que sus barcos de guerra usarían como combustible el petróleo en vez del vapor. Esta medida se debió a la aplicación del motor de combustión interna a las flotas de guerra. En consecuencia, el petróleo pasó a ser uno de los elementos taxativos para el éxito militar británico en la Primera Guerra Mundial (KLARE, 2001: 50).

No obstante, el paso de la hulla al petróleo le planteó a Londres un dilema importante. Gran Bretaña poseía ricos yacimientos de hulla en la zona correspondiente a Gales del Sur, pero dependía de la importación de petróleo extranjero ya que los recursos propios eran insignificantes. Ante la inminencia de la guerra y la eventualidad de ver comprometido el aprovisionamiento del vital suministro, el gobierno se asignó la responsabilidad directa sobre éste, justificando tal decisión bajo criterios de seguridad nacional. De esta manera, el 17 de junio de 1914 el Parlamento aprobó la adquisición pública de la participación mayoritaria en la 
Anglo Persian Oil Company ${ }^{1}$. Es dable afirmar que “(...) por primera vez la seguridad de las importaciones de petróleo se configuraba como una de las responsabilidades principales del Estado" (KLARE, 2001: 51).

Según Yergin $^{2}$ (2006: 69), desde aquella decisión de Churchill "energy security thus became a question of national strategy". En definitiva, al principio la noción de seguridad (energética) que tenían los estados se relacionaba estrictamente con la fuerza militar. Sin embargo, advertimos que en este momento histórico la unidad conceptual aún no existía como tal.

Para el realismo clásico (CARR, 1939; MORGENTHAU, 1963) el concepto de seguridad adquiere sentido en tanto el Estado, como actor principal en el sistema internacional, debe resguardar su supremacía militar en relación a los demás estados. Es decir, las amenazas a la seguridad se conciben exclusivamente de acuerdo a la habilidad de un Estado para usar la fuerza militar contra otro Estado. Por tanto, la seguridad está determinada por la capacidad militar y es concebida en términos relacionales. En definitiva, las amenazas a la seguridad de un Estado son externas al mismo.

Este modo de definir la seguridad (que ya había comenzado a tomar forma durante el período entre guerras y la Segunda Guerra Mundial) ganó significado en un contexto internacional de Guerra Fría, donde la posibilidad de guerra nuclear y destrucción masiva estaban siempre latentes. Las bases del concepto y la definición de seguridad fueron mantenidos por los exponentes del realismo estructural ${ }^{4}$ (WALTZ, 1979) hasta la caída de la cortina de hierro. Desde entonces, comenzaron a emerger en la disciplina nuevos abordajes sobre la seguridad en relación al nuevo contexto mundial imperante, que retomaremos a continuación.

Ahora bien, fueron los eventos ocurridos en Medio Oriente, en particular el primer shock petrolero en 1973, que impactarán en la conceptualización de la seguridad energética. La "securitización" del comercio de energía comenzó a partir de aquellos sucesos (BELYI, 2014: $10)$.

\section{Seguridad energética. El abordaje económico}

El 6 octubre de 1973 estalló la IV guerra árabe-israelí, también conocida como guerra del Yom Kipur o guerra del Ramadán. El hecho de importancia fundamental en este conflicto fue la apertura de un nuevo frente de batalla, muy lejos de la contienda armada: la llamada "guerra del petróleo". Los países árabes se dispusieron a "castigar" a todos aquellos estados que ayudaran a Israel, con un arma que resultó de una efectividad tal que tuvo como consecuencia la primera crisis energética mundial, cuyos efectos desestabilizaron toda la economía internacional ${ }^{5}$.

Las medidas tomadas por la Organización de Países Exportadores de Petróleo (OPEP) y la Organización de Países Árabes Exportadores de Petróleo (OPAEP) provocaron carestías en muchas regiones llevando a una reducción de la producción industrial y conduciendo a una larga

\footnotetext{
${ }^{1}$ La compañía británica de capitales privados Anglo-Persian Oil Company (APOC) se instaló en Oriente Medio desde 1901 y pasó a control del gobierno británico a partir de 1914. A partir de 1954, se constituyó en British Petroleum.

${ }^{2}$ Reconocido investigador de la temática energética, asesor político y presidente de Cambridge Energy Research Associates.

${ }_{4}^{3}$ Principales exponentes de la teoría realista clásica de las Relaciones Internacionales.

${ }^{4}$ El realismo estructural o neorrealismo es la segunda innovación en la interpretación de los postulados del realismo clásico de las Relaciones Internacionales.

${ }^{5}$ De esta manera, los países de la OPEP reunidos en Viena, decidieron implementar un aumento del $70 \%$ al precio del petróleo. Por su parte, la OPAEP (Organización de Países Árabes Productores de Petróleo) reunida en Kuwait, decidió disminuir la producción de petróleo en un 5\% mensual acumulativo hasta que Israel se retirara de los territorios ocupados desde 1967. Seguidamente, declararon un embargo petrolero de seis meses a Estados Unidos y Holanda -que se amplió a Portugal, Rhodesia (hoy, Zambia y Zimbabue) y Sudáfrica (GATTI, 1992: 46).
} 
recesión económica. Fue a partir de este momento cuando el petróleo, que ya era un factor esencial desde el punto de vista militar, alcanzó la consideración de requisito indispensable para la estabilidad económica mundial (KLARE, 2001:54).

A partir del primer shock petrolero, los temas energéticos pasaron a ser prioritarios en el contexto internacional. Tal es así que la reacción inmediata de los países industrializados fue la creación en 1974 de la Agencia Internacional de la Energía (AIE), organismo vinculado a la Organización para la Cooperación y el Desarrollo Económico (OCDE). La primera decisión tomada por la AIE fue la de mantener existencias de petróleo (conocidas como reservas estratégicas de petróleo) y promover consultas regulares en vistas a garantizar una reacción coordinada ante la eventualidad de nuevas crisis energéticas (PAIVA BENEVIDES, 2011: 46).

De la coyuntura de crisis analizada se pueden esgrimir tres conclusiones parciales. Primero, surgió la unidad conceptual seguridad energética. Segundo, al inicio, la concepción de la seguridad energética estuvo vinculada exclusivamente al petróleo crudo como recurso natural no renovable. Tercero, al tratamiento geopolítico imperante se le suma un abordaje económico del concepto ${ }^{6}$, es decir, la relación entre la disponibilidad del suministro y el precio del petróleo.

Huelga decir que durante esos años se crearon las primeras agencias gubernamentales al interior de los estados más dependientes del hidrocarburo, con una dedicación exclusiva a la temática energética ${ }^{7}$. Dichos organismos tuvieron como preocupación central asegurar el suministro petrolero, y en menor medida, desarrollar energías alternativas para disminuir la dependencia de sus economías del petróleo. Más aún, la primera acta de política energética fue sancionada después del shock petrolero de 1973 en los Estados Unidos ${ }^{8}$. Si bien el término "energético" estaba relacionado con las preocupaciones relativas al petróleo, el concepto tímidamente comenzó a englobar otras sub-áreas, como las energías renovables.

En Estados Unidas, dichas primeras decisiones públicas en lo que concierne a la cuestión energética coincidió temporalmente con el cenit de la producción de petróleo en ese país. En 1956, el geofísico Marion King Hubbert había profetizando el momento en que se acabaría el petróleo en suelo norteamericano basándose en una teoría que ilustra el ritmo de descubrimiento y producción del crudo. De acuerdo a sus investigaciones, el peak oil es el punto en el tiempo en el que la producción de petróleo alcanza su pico máximo, después del cual declina hasta una eventual interrupción; lo cual permitió predecir correctamente el punto culminante del recurso en Estados Unidos en 1971 (ALEKLETT ET AL., 2010: 20). Para autores como García Reyes y Lozada García (2015) este factor sumado al shock petrolero dio nacimiento al término seguridad energética en Estados Unidos.

\footnotetext{
${ }^{6}$ El caso de Alemania ayuda a ilustrar dicha afirmación ya que en ese momento las temáticas energéticas eran abordadas por el Ministerio de Economía. Recién en el año 2005, el Ministerio fue renombrado Ministerio Federal de Asuntos Económicos y Energía (Federal Minister of Economic Affairs and Energy).

${ }^{7}$ En este sentido, y a modo de ejemplo, vale mencionar que la Oficina de la Energía Fósil (Office of Fossil Energy), que data de 1909 era el organismo encargado de la regulación interna de la producción de petróleo en Estados Unidos. Con el primer shock petrolero, se reorganizó la estructura gubernamental interna, creándose en 1977 el Departamento de Energía (DOE, por sus siglas en inglés) con diferentes subsecretarías, incluyendo la anteriormente mencionada. Es importante dar a conocer que desde 1942 el DOE había tenido dedicación exclusiva al desarrollo de la tecnología nuclear. Finalmente, importa resaltar que en 1974 se creó la Administración de Información de la Energía de Estados Unidos (United States Energy Information Administration), en respuesta a la necesidad del gobierno federal de recolectar información relativa a la energía.

Por su parte, en Gran Bretaña se estableció en 1974 el primer departamento de energía y en Japón se instituyó la Agencia para Recursos Naturales y Energía (Agency for Natural Resources and Energy) en 1973.

8 En 1975, el entonces Presidente Ford firmó la primera legislación sobre política energética y conservación (Energy Policy and Conservation Act) no solamente abocada a crear una reserva de emergencia en caso de escasez mundial de petróleo sino con el fin de lograr una mayor inversión en investigación y desarrollo de energías alternativas.
} 
Podemos observar una tendencia a unificar los temas relativos al aprovisionamiento energético en la diagramación de las políticas públicas. En otras palabras, el término "energía" pasó entonces a nuclear el tratamiento de todas las fuentes de recursos energéticos (petróleo y gas, atómica, biocombustibles, de recursos renovables, etc.).

Es interesante observar que ante las problemáticas consecuencias que trajo consigo el shock petrolero de 1973, los mayores importadores de petróleo a nivel mundial aglutinados en la AIE comenzaron a considerar a la seguridad energética desde una visión economicista, definiéndola como "suministro confiable a precios razonables" . No obstante, para los países exportadores de petróleo reunidos en la OPEP, el concepto supuso otro significado: mantener la "seguridad de la demanda" para conservar ingresos altos en las arcas gubernamentales (YERGIN, 2006: 70-71).

Estudios posteriores, como los de Susan Strange, realizaron un acercamiento al concepto aquí analizado justamente desde la Economía Política Internacional. La autora afirmó en su libro States and Markets (1980) que el shock petrolero de 1973 fue provocado parcialmente por la guerra árabe-israelí, por tanto no puede ser analizado solamente en términos cuantitativos (considerando la ecuación escasez de suministro/precios altos). Como exponente del realismo estructural, argumentó que las cuatro estructuras primarias, a saber, seguridad, finanzas, producción y conocimiento, constituyen las causas del poder estructural de los actores internacionales. Entonces, la energía juega un rol vital para la producción (especialmente, para la industria, el transporte y el sector urbano-residencial), para las finanzas (en cuanto a los beneficios que provee el mercado del petróleo), para el conocimiento (en lo referente al desarrollo tecnológico, incluido también el sector medioambiental) y para la seguridad (en lo que hace al establecimiento de instituciones que se ocupen del suministro energético o la intervención militar en regiones productoras de petróleo) (STRANGE, 1980).

Por tanto, después del segundo shock petrolero de 1979, el tratamiento del concepto empieza a beneficiarse de otros exámenes multidisciplinares, que se sumaron a los primigenios estudios relativos a la seguridad considerada como seguridad nacional primero y como comportamiento del mercado después.

\section{Seguridad energética. Hacia un concepto multidimensional}

El fin de la Guerra Fría trajo consigo un nuevo contexto internacional que pasó a tener incidencia directa sobre los estudios de seguridad y de seguridad energética. El afianzamiento de un sistema unipolar bajo el liderazgo de Estados Unidos durante los años 90' abrió determinados espacios que antes se encontraban dominados bajo la lógica Este-Oeste. En consecuencia, la agenda internacional comenzó a ser definida por "nuevos temas", tales como la defensa de los Derechos Humanos, la lucha contra la pobreza mundial, el narcotráfico o las preocupaciones medioambientales.

Estas nuevas amenazas ya no podían ser tratadas solamente por las políticas de defensa tradicionales, es decir, a través de la utilización de los recursos de poder duro ${ }^{10}$, en términos de Joseph Nye (1990). Por el contrario, se debieron buscar nuevas formas de contrarrestarlas, pues en la mayoría de los casos la utilización de la fuerza no se consideraba la herramienta más útil o eficaz (CUJABANTE, 2009: 101).

Bajo tal contexto histórico, surgieron los estudios críticos sobre la temática de seguridad. El mayor exponente de tal corriente fue Barry Buzan quien se unió a la Escuela de Copenhague a principios de 1990, redefiniendo tal concepto. Cabe dar a conocer que aquella Escuela heredó la perspectiva realista de las Relaciones Internacionales, por tanto su producción tuvo una impronta muy importante de aquella teoría. Lo anterior se vuelve tangible ya que se

\footnotetext{
${ }^{9}$ Datos extraídos de la página web de la AIE http://www.iea.org/topics/energysecurity/ Consulta: 24 de noviembre de 2016.

${ }^{10}$ Según Joseph Nye, el poder duro (hard power) es el poder militar y el poder blando (soft power) es la habilidad de un Estado para persuadir otros estados sin el uso de medios coercitivos.
} 
siguió considerando a la anarquía como la característica que define la estructura internacional, la cual también explica las actuaciones del Estado de acuerdo a su percepción de (in)seguridad.

De modo que en aquellos desarrollos teóricos ${ }^{11}$, el término seguridad incluía cinco variables o sectores con centro en el Estado: el político -que abarca la estabilidad interna y externa del Estado-; el militar -que cubre las capacidades defensivas y ofensivas-; el social -que significa la estabilidad de la identidad cultural, por ejemplo nacional o religiosa-; el económico -relacionado al acceso de recursos y mercados- y el medioambiental -definido como la protección de la biósfera (BUZAN, 1991: 19).

A pesar que la Escuela de Copenhague no distinguió a la seguridad energética, ésta se evidencia en las variables anteriormente consideradas. En primer lugar, la seguridad política en las Relaciones Internacionales se refiere a las relaciones de seguridad que se establecen con otros estados y que son determinadas por un sistema internacional anárquico. En este sentido, los estados buscan asegurar su autosuficiencia energética para dejar de depender del suministro externo. Si un país es importador neto de recursos naturales estratégicos (petróleo, gas, carbón, etc.), la interrupción del suministro es una amenaza siempre latente. Por ello, establecen alianzas políticas como la que Estados Unidos mantiene con Arabia Saudita.

En segundo lugar, la disponibilidad de energía tiene influencia directa sobre las capacidades militares, las cuales para su despliegue dependen en gran medida de recursos naturales estratégicos.

En tercer lugar, la seguridad económica adquiere sentido frente a la dificultad de predecir el comportamiento de los actores económicos de una economía capitalista descentralizada (BUZAN, 1991: 235-237). Entonces, la securitización de la energía está relacionada al accionar político de los estados con el objetivo de menguar la imprevisibilidad del mercado energético.

Finalmente, en cuarto lugar, la seguridad medioambiental supone la incompatibilidad entre el desarrollo económico acelerado y la protección de los recursos naturales. En 1970, esta problemática estaba centrada en la preocupación por el agotamiento de los combustibles fósiles, especialmente el petróleo. Mientras que en la década del 90' la seguridad ambiental se tradujo en la concepción del cambio climático como una amenaza con efectos globales debido a las emisiones de carbono que genera el uso creciente de los combustibles fósiles.

Al respecto, las variables social y ambiental deben ser reflexionadas a la luz de los acontecimientos de la época. Los años 90' vieron surgir las preocupaciones vinculadas al medio ambiente que se plasmaron en la Conferencia de las Naciones Unidas sobre el Medio Ambiente y el Desarrollo, en Rio de Janeiro en 1992. Una de las razones que hizo de esta conferencia el encuentro más importante de todos los tiempos en la materia fue, no sólo la gran asistencia de jefes de Estado (cerca de 120) y de medios de comunicación sino, la participación de organizaciones de la sociedad civil, que ya desde el período de preparación aportaron ideas para que sus preocupaciones fueran atendidas en esta reunión (BUENO, 2009).

De la declaración homóloga surgió el concepto de desarrollo sostenible y también el principio de 'las responsabilidades comunes pero diferenciadas', que resumía el reconocimiento de los países industrializados de su mayor responsabilidad en la degradación ambiental, lo cual

\footnotetext{
${ }^{11}$ Particularmente, hay dos obras que plasmaron las nuevas preocupaciones en torno al concepto de seguridad durante la década de 1990, a saber: People, States and Fear: an agenda for international security studies in the post-cold war era de BUZAN, B. (1991) y Security: A New Framework for Analysis de BUZAN, B.; WÆVER, O., DE WILDE, J. (1998).
} 
habilitó el compromiso asumido en el Protocolo de Kyoto ${ }^{12}$ firmado cinco años después (BUENO, 2009).

En otras palabras, la reconceptualización de la seguridad planteada por la Escuela de Copenhague sintetizó lo que transcurrió durante aquellos momentos, sumando las variables social y ambiental al concepto, además de las variables político-militar y económica que ya poseía. De este modo, junto a aquellos desarrollos teóricos emergió una aproximación holística del concepto.

También se puede encontrar un cierto sesgo constructivista en los abordajes de la seguridad de Barry Buzan. Esto se hace evidente al considerar junto con el autor que "la definición de securitización está constituida por el establecimiento intersubjetivo de una amenaza existencial, con una importancia suficiente para tener efectos políticos" (BUZAN ET AL., 1998: 25). Por tanto, no solamente el Estado es central en su forma de analizar la seguridad, sino también la sociedad y el individuo y cómo la misma sociedad construye o securitiza las amenazas.

En el apartado siguiente se expondrá la manera en que los acontecimientos del 11 de septiembre de 2001 marcaron un retroceso en la forma de concebir la seguridad en las políticas internas y externas de los estados. Sin embargo, el mayor protagonismo internacional de potencias emergentes como India, China y Brasil terminó afianzando el concepto de seguridad energética a finales de la primera década del nuevo milenio.

\section{La seguridad energética desde 2001 al presente}

Los atentados al World Trade Center y al Pentágono el 11 de septiembre de 2001 significaron un punto de ruptura para los Estados Unidos y para las Relaciones Internacionales. La inmediata reacción de la mayor potencia mundial no tardó en llegar, materializándose en las intervenciones en Afganistán primero y en Irak después, que marcaron el inicio de la guerra contra el terrorismo y securitización de la agenda internacional.

La seguridad, entonces, volvió a estar vinculada directamente con la seguridad nacional tal como era concebida desde el realismo. El poder, traducido en capacidad militar, pasó nuevamente al centro de la escena en las relaciones entre los estados. No obstante, la percepción de la amenaza dejó de ser concebida exclusivamente por fuera del Estado. Los atentados del 11$\mathrm{S}$ fueron la piedra de toque para que se concibiera al terrorismo como un fenómeno transnacionalizado, que permea las fronteras estatales como consecuencia directa de la globalización.

En definitiva, las amenazas comenzaron a contemplarse como cada vez más numerosas e interrelacionadas, con un doble anclaje tanto doméstico como internacional, esferas que a su vez se retroalimentan. El terrorismo internacional, entonces, sería producto de la vulnerabilidad que generan los altos índices de pobreza, el deterioro de las instituciones gubernamentales y la falta de desarrollo económico en determinados países. Bajo dicha interpretación, estas causas pasaron a conformar el ambiente en el que se constituirían las células terroristas y también formaron parte de las justificaciones de Washington para encabezar la defensa de la democracia y los Derechos Humanos en Medio Oriente.

Este nuevo contexto internacional fue teorizado por la tercera innovación de los postulados realistas. Mientras los teóricos del realismo clásico y los neorrealistas continuaban haciendo foco en que las amenazas emanaban desde fuera de las fronteras del Estado soberano,

\footnotetext{
${ }^{12}$ Tratado internacional de limitación de emisiones de dióxido de carbono (CO2) firmado en 1997. Dicho protocolo entró en vigor diez años después, fijando límites para las emisiones de Gases de Efecto Invernadero (GEI) de los países industrializados. Estados Unidos, en ese momento el primer emisor de GEIs del mundo, no sólo no ratificó el documento sino que bajo la administración de George W. Bush retiró la firma.
} 
el realismo neoclásico ${ }^{13}$ comenzó a enfatizar tanto en las fuentes internas como externas de las amenazas (SCHMIDT, 2008: 157). En este sentido, desde el realismo neoclásico se puede vislumbrar que, en palabras de Melvyn Leffler, la seguridad se define como la protección de valores centrales domésticos de las amenazas externas, en tanto sólo emergen en relación a cómo un Estado define sus ejes conductores internos. Así, es posible ofrecer un enfoque de la seguridad que incorpore tanto la política doméstica como el sistema internacional (SCHMIDT, 2008: 158).

Para poder explicar este nuevo enfoque interméstico de la seguridad, el realismo neoclásico tomó distancia del realismo estructural al afirmar que no existe necesariamente una correa de transmisión perfecta entre los incentivos del sistema y las políticas llevadas a cabo por los gobiernos. Si bien afirma que la posición en el sistema internacional y las capacidades materiales relativas de un Estado siguen siendo determinantes para comprender su política exterior, reconoce un conjunto de variables intervinientes que ejercen singular influencia en el proceso de toma de decisiones, como la percepción de los líderes y la estructura doméstica. De este modo, la innovación del realismo neoclásico se centró en articular variables del nivel internacional con variables del nivel doméstico para explicar las razones por las cuales estados con capacidades materiales relativas similares actúan diferente ante iguales circunstancias (ROSE, 1998).

Ahora bien, a pesar del giro en la política exterior norteamericana a partir del 11-S y los cambios que condujo en la forma de percibir y construir la seguridad a nivel mundial, cabe considerar que durante los primeros meses de la administración de George W. Bush, el vicepresidente Dick Cheney presentó una propuesta referida a la seguridad energética del país dada la intensa dependencia del suministro petrolero externo. Si bien el informe fue preparado por el Grupo de Desarrollo de Política Energética Nacional (National Energy Policy Develoment Group), se lo denominó 'informe Cheney' y fue motivo de numerosas polémicas. En cuanto a las implicaciones internacionales de la política energética, se consideraba que el creciente nivel de consumo de petróleo en Estados Unidos sólo podía mantenerse si los proveedores extranjeros incrementaban su producción y vendían más cantidad de crudo al país. Tal es así que para poder satisfacer dichas necesidades energéticas recomendó a la Casa Blanca asegurar las importaciones petroleras y considerarlas como prioridad de la política comercial y exterior, basándose en dos objetivos. El primero consistía en aumentar las importaciones procedentes de los países del Golfo Pérsico y el segundo buscaba aumentar la diversidad geográfica de las importaciones del país del Norte, a fin de reducir las consecuencias económicas de futuros conflictos en una región permanentemente inestable (RUIZ CARO, 2007: 47-48).

No obstante, en 2005 se firmó un Acta de Política Energética que constituyó un cambio en la prioridad del enfoque para lograr la seguridad energética en Washington, debido a que el gobierno le otorgó un mayor énfasis a la reducción de la dependencia de recursos energéticos de origen fósil. En la Iniciativa Energética Avanzada presentada al Consejo Económico Nacional en 2006, la administración republicana consideró un incremento de $22 \%$ en fondos para la investigación de tecnologías limpias (RUIZ CARO, 2007: 48).

En consecuencia de lo anterior, importa remarcar dos cuestiones de importancia. Por un lado, que a pesar del mencionado rechazo al Protocolo de Kyoto por la administración de George W. Bush, al interior de Estados Unidos se vislumbró cierto sesgo de cambio en cuanto a la forma de concebir la seguridad energética de acuerdo al patrón de consumo basado en hidrocarburos, dadas las consecuencias medioambientales que ello conlleva. Por otro lado, ya se comenzaban a escuchar críticas al gobierno respecto de las 'verdaderas intenciones' de la invasión a Irak, al haber fracasado la operación que justificaba dicho accionar en las supuestas armas de destrucción masiva que no fueron halladas. Desde distintos rincones del mundo se relacionaba la capacidad de asegurar su suministro petrolero al uso del poder duro norteamericano, ideas que ya habían sido anticipadas en el Proyecto para el Nuevo Siglo

\footnotetext{
${ }^{13}$ El realismo neoclásico fue definido por primera vez por Gideon Rose en 1998 en el artículo "Realismo Neoclásico y teorías de política exterior".
} 
Americano de 1998, emitido por un grupo de ultraconservadores que ahora formaban parte del staff político en el poder (BUSSO, 2003).

Avanzada la primera década del nuevo milenio, una secuencia de hechos relevantes volvió a traer a escena el concepto de seguridad fuertemente atado a la energía: el denominado tercer shock petrolero como un nuevo factor disruptivo; el cambio de administración en la Casa Blanca y la aplicación de su 'poder energético' como herramienta de política exterior y el creciente protagonismo de países definidos como potencias emergentes (principalmente China, Brasil e India) y el altísimo consumo energético que mostraban.

Por una parte, entre los años 2003 y 2008, se registró un nuevo ciclo de elevación de precios del barril de crudo, motivado por una serie de factores: la declinación de la producción en regiones no controladas por la OPEP (como el Golfo de México y el Mar del Norte), el crecimiento de la economía mundial que impulsó hacia arriba la demanda de petróleo, la recuperación del poder de mercado de la OPEP (debido al aumento del precio del commodity) y la inestabilidad política en Medio Oriente, zona que guarda más del $60 \%$ de las reservas mundiales del hidrocarburo. En base a dichos factores, hay cierto consenso en afirmar que el sistema internacional pasó por un tercer shock petrolero en la primera década del siglo XXI.

Cabe reflexionar que los efectos negativos del tercer shock petrolero se combinaron con la crisis financiera internacional de 2008, acentuando la recesión económica principalmente en Estados Unidos y la Unión Europea. Dicho impacto se pudo observar en las oscilaciones en el precio del barril de petróleo, el cual en julio de 2008 alcanzó los 140 dólares en la bolsa de Nueva York, para luego decaer en el segundo semestre de ese año y volver a subir en el primer semestre de 2009, dados los primeros signos de recuperación económica de los países occidentales (PAIVA BENEVIDES, 2011: 48).

Por otra parte, la elección de Barack Obama como presidente de Estados Unidos y su inmediato anuncio de retirar las tropas de Afganistán modificó la agenda internacional. Si bien las temáticas de seguridad siguieron vigentes, hubo una disminución en la intensidad en comparación con la anterior administración republicana. Sin embargo, autores como Klare (2015) advirtieron que en la consecución de sus objetivos de política exterior, la administración demócrata en vez de optar por hacer uso de su poder duro o su poder blando, usufructuó su 'poder energético', definido como "the exploitation of a nation's advantages in energy output and technology to promote its global interests and undermine those of its rivals". Como consecuencia del desgaste de Estados Unidos en su despliegue militar en Medio Oriente así como los reclamos al nuevo gobierno por un cambio respecto de dicha política exterior, el escenario que se presentaba daba la oportunidad para poner a prueba el poder energético como una alternativa viable al poder duro, en un momento en que el uso de la fuerza militar aparecía como poco deseable (KLARE, 2015).

La pregunta que cabe hacerse aquí es como pudo llevarse a cabo tal despliegue de poder energético. La respuesta debe encontrarse en el boom del fracking ${ }^{14}$ que tuvo lugar en Estados Unidos a partir de 2010 y que fue analizado como una forma de reducir la dependencia del petróleo y del gas de Medio Oriente, otorgándole un mayor margen de maniobra en dicha región. El país del Norte pasó de producir 5,6 millones de barriles diarios de petróleo en 2010 a 9,3 millones a fines de 2014, el mayor nivel en 30 años (BELLATO, 2015). Un ejemplo que puede ilustrar el uso de tal herramienta de política exterior lo expusieron públicamente dos senadores republicanos, John McCain y John Hoeven en un artículo publicado en 2014 en el Wall Street Journal demostrando que la venta de gas natural producido en Estados Unidos debería convertirse en una forma de afianzar lazos con los aliados europeos $\mathrm{y}$, a su vez, debilitar el régimen de Vladimir Putin (KLARE, 2015).

Frente al reciente cambio de gobierno en Estados Unidos, será interesante ponderar la mencionada postura republicana bajo la política energética de la era Trump, quien ha afirmado que su objetivo será independizar al país por completo del suministro exterior.

${ }^{14}$ El fracking, o la fractura hidráulica, es una técnica utilizada en la industria petrolera para extraer petróleo y gas no convencionales. 
En último lugar, desde que en 2001 salió a la luz el acrónimo BRIC acuñado por la entidad financiera Goldman Sachs, Brasil, India y China principalmente pasaron a estar en el centro de la escena mundial. Los altos niveles de crecimiento económico sostenido y el reconocimiento internacional a su desempeño económico positivo, derivaron en un mayor sentimiento de confianza de las elites de gobierno de estos tres países teniendo como consecuencia un mayor activismo internacional.

Sin embargo, en un mundo regido por un patrón de crecimiento basado en el consumo de hidrocarburos, el aumento exponencial de la demanda de petróleo de China y de India supuso una preocupación cada vez mayor para los países industrializados importadores netos de crudo. Particularmente, el peso específico de China en el mercado energético es enorme ya que el nuevo siglo la vio convertirse en el mayor consumidor y productor de energía del mundo. Actualmente es el mayor consumidor de petróleo, considerando que en 2014 pasó a ser el primer importador de este recurso a nivel mundial. De acuerdo a la base 'business-as-usual' (según los parámetros de consumo actuales, sin aplicársele cambio alguno) se pronostica que Beijing se convertirá en el principal consumidor de petróleo en 2030 superando así a Estados Unidos. India, por su parte, contribuye con la mayor proporción de crecimiento de la demanda energética mundial, siendo el tercer mayor consumidor de energía, detrás de China y Estados Unidos. Además, es el cuarto mayor consumidor de petróleo a nivel mundial, dependiendo en un $80 \%$ del aprovisionamiento externo y se proyecta que será el principal importador de carbón para el 2020 (DUSSORT, 2016).

En este contexto, la posibilidad de rivalidades geopolíticas por los recursos energéticos -tanto dentro de Asia como entre Asia y Occidente- así como el diseño de estrategias para asegurar el suministro es una realidad que está sustentada fundamentalmente en dos hechos. En primer lugar, las intensas negociaciones por los gasoductos y oleoductos procedentes de Oriente Medio, Asia Central y Rusia entre asiáticos, por un lado, y europeos y norteamericanos por el otro. En segundo lugar, el reciente aumento de las inversiones de empresas públicas gasíferas y petroleras de Asia en regiones productoras de todo el mundo, a menudo en cooperación con las empresas estatales de esos mismos países productores, creando competencia no sólo entre las empresas asiáticas sino también entre las empresas estatales en general (las empresas petroleras nacionales) y las empresas privadas de los países consumidores de Occidente (las empresas petroleras internacionales) (RUIZ CARO, 2007: 59-60).

Ante los factores mencionados en los párrafos anteriores, el concepto de seguridad volvió a estar muy vinculado al acceso al petróleo como recurso estratégico, aunque cabe considerar que las cuestiones medioambientales ya habían ganado su lugar en la agenda y, por tanto, no debe entenderse la seguridad energética sin esta variable. Es decir, que se puede presumir que no hubo retroceso desde que el concepto ha tenido un abordaje multidimensional, lo cual está presente en los esfuerzos de los países industrializados como en las potencias emergentes por modificar la matriz energética mundial y hacerla más sustentable. Al respecto es necesario mencionar el acuerdo histórico alcanzado en el año 2015 en la Conferencia de Naciones Unidas sobre Cambio Climático reunida en París (conocida como COP21), en donde los compromisos asumidos por Brasil, India, China y Sudáfrica (BASIC) tuvieron un rol protagónico para alcanzar dicho acuerdo con el fin de aminorar las emisiones de gases tóxicos a la atmósfera que provocan el cambio climático ${ }^{15}$.

Resta decir que este estado de evolución del concepto de seguridad energética se puede ver claramente en las últimas publicaciones de la Agencia Internacional de la Energía, como organismo internacional más representativo en materia energética. Allí se reconoce que, desde sus inicios, la organización se había propuesto garantizar la seguridad energética entendida como la seguridad en el aprovisionamiento de petróleo, tal como se mencionó en los anteriores párrafos. Empero, mientras afirma que asegurar el suministro de petróleo continúa siendo

15 "La Cumbre de París logró cerrar un acuerdo histórico por el cual todos los países firmantes se comprometieron a limitar sus emisiones. De este modo, se dejó de lado la disputa sobre la diferenciación abarcando a todos los signatarios. El texto estableció, empero, mayores exigencias para los países desarrollados quienes deberán 'seguir encabezando los esfuerzos"” (GIACCAGLIA, 2017: 449). 
crucial, las políticas de seguridad energética contemporáneas necesariamente tienen que abordar todas las fuentes de recursos así como cubrir un rango de riesgos políticos, económicos y naturales que afectan a la infraestructura y los servicios. Por ello, se requiere de análisis comprensivos de la seguridad energética nacional para poder esbozar la política energética adecuada (AIE, 2011: 6).

De esta forma, la AIE ha desarrollado un Modelo de Seguridad Energética de Corto Plazo (MOSES, por sus siglas en inglés). Éste ha sido utilizado para analizar siete fuentes primarias del suministro de energía (petróleo crudo, gas natural, carbón, bioenergía, energía hidroeléctrica, energía geotermal y energía nuclear) y dos grupos de fuentes secundarias (derivados de petróleo y biocombustibles). Además, aborda dos dimensiones de la seguridad energética: la externa, que incluye la importación de energía, y la doméstica, que abarca factores relacionados a la producción, la distribución y la transformación de la energía. Las dimensiones doméstica y externa reflejan los riesgos y la adaptación ante una interrupción en el suministro (AIE, 2011: 9-10).

Lo novedoso de esta nueva forma de aproximarse al concepto de seguridad energética se debe a que se agregó la variable tecnológica a cada una de las concepciones ya desarrolladas. La producción y la transformación de la energía tienen que ver básicamente con los avances tecnológicos y técnicos que vuelven ciertos recursos económicamente explotables. A pesar que el presente modelo es de corto plazo, los análisis de la seguridad energética de largo plazo también observan indicadores relativos al impacto ambiental, el crecimiento de la demanda energética, el agotamiento de los recursos naturales y la volatilidad de los precios de la energía. Con estas mejoras teóricas, la AIE ha reconocido que la dependencia en la importación de petróleo de un determinado país, los stocks de emergencia y la diversificación de proveedores no son suficientes para entender la seguridad energética.

Finalmente, es preciso señalar que las instituciones nacionales e internacionales que fueron creadas para promover la seguridad energética desde el primer shock petrolero hasta la actualidad están luchando por permanecer en escena. Como se dijo, la AIE es el organismo internacional más importante en cuestiones relativas a energía pero ha hecho pocos esfuerzos para involucrar a los 'nuevos gigantes' consumidores de energía en su proceso de toma de decisiones. Es decir, cuando la AIE coordina respuestas frente a una crisis energética, los jugadores más importantes en cuanto a consumo (a saber, India y China) y producción de petróleo no tienen voz. Y por el momento los miembros actuales no tienen intenciones de hacer de la organización un foro menos restringido ya que pondría en jaque sus propias posiciones de poder (VICTOR; YUEH, 2010).

En tanto, la gobernanza mundial de la política energética presenta un panorama fracturado que puede resumirse en dos bandos contrapuestos: por un lado, los países de la OCDE reunidos en la AIE como grandes consumidores y por otro lado, la OPEP que reúne a los mayores productores de petróleo. Si bien en el transcurso de los años han surgido otras organizaciones como el Consejo Mundial de la Energía, que nuclea a gran parte de los países que no conforman a aquellas dos organizaciones internacionales, no posee un gran poder de influencia en el contexto energético mundial sino más bien funciones consultivas.

De acuerdo a autores como Victor y Yueh (2010) cualquier esfuerzo por coordinar una política energética global debe incluir a los 'jugadores más importantes', aunque ello presuponga establecer consensos comunes sobre una única concepción de seguridad energética, que englobaría intereses dispares. Tal y como se ha reparado, la seguridad energética de Estados Unidos está fuertemente definida por su dependencia/no dependencia del petróleo extranjero. En cuanto a la Unión Europea, la propia Comisión Europea ha declarado que la energía debe convertirse en un elemento clave de todas las relaciones exteriores y que se trata de un factor crucial para la seguridad geopolítica, la estabilidad económica, el desarrollo social y los esfuerzos internacionales en la lucha contra el cambio climático (RUIZ CARO, 2007: 56).

Para el caso de las potencias emergentes como China e India, sus concepciones de la seguridad energética estaban centradas en la adquisición de los hidrocarburos necesarios para mantener sus niveles de crecimiento económico, que desde la COP 2015 se han comprometido 
en que ese crecimiento sea 'sustentable'. En lo que respecta a la región latinoamericana, la seguridad energética es un concepto foráneo por la abundancia de recursos naturales estratégicos, aunque viene siendo relacionada a la mitigación del cambio climático y a la necesidad de integración energética; cuestiones que han sido discutidas en organizaciones tales como la Organización Latinoamericana de Energía (OLADE) y la Comisión Económica para América Latina y el Caribe (RUIZ CARO, 2007).

A pesar de la evolución que ha tenido el concepto aquí analizado, la falta de consenso internacional nos conduce a la imagen de que la creación de un régimen internacional en materia energética se ve aún como un horizonte lejano.

\section{Reflexiones finales}

El presente trabajo ha intentado ser un ejercicio de aplicación del género historia conceptual desarrollado por Reinhart Koselleck al concepto de seguridad energética. En los anteriores apartados se analizaron las modificaciones que fue sufriendo la definición del concepto a la luz de los acontecimientos del escenario internacional.

En este ejercicio de aplicación han quedado claras dos cuestiones respecto de la conformación del concepto de seguridad energética. En primer lugar, que la unidad conceptual surge producto de una situación de crisis a nivel internacional que deriva en la unión de dos conceptos anteriormente constituidos: seguridad, hasta ese momento relacionado a la capacidad militar y energía, concebida exclusivamente como petróleo.

En segundo lugar, a partir de la conformación de la unidad conceptual, el significante que le dio contenido al concepto de seguridad energética fue mutando hasta alcanzar una definición que reflejó la evolución de la agenda internacional. En consecuencia, el shock petrolero de 1973, le dio su impronta económica; las conferencias sobre el medioambiente, le concedieron su veta ambiental y social y el tercer shock petrolero, le sumó el desarrollo tecnológico así como amplió las fuentes de abastecimiento de energía.

Retomando, se puede ver entonces que en la actualidad la seguridad energética ha pasado a tener un abordaje multidimensional. El concepto surgió relacionado exclusivamente al petróleo. Después fue transformándose hasta convertirse en un concepto más holístico, que incluyó de forma agregada las diferentes dimensiones por las que ha atravesado: seguridad nacional (político-militar), económica, ambiental, social y tecnológica.

De esta manera, se puede concluir que de las cuatro posibilidades que existen para analizar el cambio recíproco de los conceptos y las circunstancias que Koselleck atribuye, la que mejor se adapta es aquella que esboza que el significado de la palabra, así como el de las circunstancias aprehendidas en ella permanecen sincrónica y diacrónicamente constantes. En otras palabras, hubo continuidades en el concepto primigenio de seguridad, que se atribuía a lo político-militar, hubo modificaciones, como se explicó en el párrafo anterior y también hubo alteraciones en el uso.

Se puede deducir del estudio anterior que el surgimiento del concepto de seguridad energética como una unidad conceptual fue contemporáneo al advenimiento de la sanción de las primeras políticas energéticas al interior de los estados. Aquellas políticas públicas recogieron el concepto de seguridad energética con la impronta de cada época histórica. En tanto, sufrieron las mismas transformaciones o adaptaciones.

En resumidas cuentas, se puede concluir que hubo un suceder de circunstancias que fueron objetivadas en el significante del concepto de seguridad energética a lo largo del tiempo, dando como resultado un concepto multidimensional. 


\section{Bibliografía}

ALEKLETT, K., KHÖÖK, M., JAKOBSSON, K., LARDELLI, M., SNOWDEN, S., SÖDERBERGH, B. (2010). The Peak of the Oil Age. Analyzing the world oil production, Reference Scenario en World Energy Outlook 2008, Energy Policy, London.

BELLATO, R. (2015). El precio del petróleo afecta al 'fracking' en Estados Unidos en RT en Español, 18 de marzo de 2015, Moscú. Disponible en: https://actualidad.rt.com/opinion/roberto-bellato/169450precio-petroleo-afecta-fracking-estados Consulta: 4 de mayo de 2017 Consulta: 4 de mayo de 2017.

BELYI, A. V. (2014). Energy security in International Relations (IR) theories, Higher School of Economics, Moscú.

BUENO, M. del P. (2009). La cuestión ambiental desde la óptica latinoamericana, Facultad de Ciencia Política y Relaciones Internacionales, Universidad Nacional de Rosario.

BUSSO, A. (2003). La política exterior americana a partir de la administración de George W. Bush: su impacto mundial y regional, Conferencia: La política exterior americana a partir de la administración de George W. Bush: su impacto mundial y regional; Universidad Torcuato Di Tella, Buenos Aires.

BUZAN, B. (1991). People, States and Fear: an agenda for international security studies in the post-cold war era, Harvester Wheatsheaf, Londres.

BUZAN, B., WAEVER, O., DE WILDE, J. (1998). Security: A New Framework for Analysis, Lynne Rienner Publishers, Boulder.

CARR, E. H. (1939). The Twenty Years Crisis, 1919-1939: an Introduction to the Study of International Relations, Macmillan, Londres.

CUJABANTE, X. (2009). La seguridad internacional: Evolución de un concepto en Revista de Relaciones Internacionales, Estrategia y Seguridad, 4 (2), Universidad Militar Nueva Granada, Colombia.

DUSSORT, M. N. (2016). La seguridad energética para los gigantes asiáticos en Voces en el Fénix, Plan Fénix, Facultad de Ciencias Económicas, Universidad de Buenos Aires.

GARCÍA REYES, M.; LOZADA GARCÍA, A. (2015). La seguridad energética en América Latina hasta el 2015, Fundación Konrand Adenauer, Rio de Janerio.

GATTI, L. (1992). El conflicto árabe-israelí: desde sus orígenes hasta los acuerdos de Camp David en Cuadernos de Política Exterior, Serie Docencia 15, CERIR, Rosario.

GIACCAGLIA, C. (2017). Poderes tradicionales, emergentes y reemergentes: relaciones ambiguas pero pragmáticas en Foro Internacional, LVII (2), Colegio de México.

JEWELL, J. (2011). The IEA Model of Short-term Energy Security (MOSES) Primary Energy Sources and Secondary Fuels, International Energy Agency.

KLARE, M. T. (2001). Guerras por los recursos. El futuro escenario del conflicto global, Ediciones Urano, Barcelona.

KLARE, M. T. (2015). Hard power, soft power, and energy power en Foreign Affairs, snapshot, March 3.

KOSELLECK, R. (2004). Historia de los conceptos y conceptos de historia en Ayer, № 53.

LESGART, C. (2001). Innovación Conceptual y Cambio Político en Revista Argentina de Ciencia Política, Buenos Aires.

LOBELL, S. E., RIPSMAN, N. M. y TALIAFERRO, J. (eds.) (2009). Neoclasical realism, the state and foreign policy, Cambridge University Press, Cambridge.

MORGENTHAU, H. (1963). Política entre las nacionales. La lucha por el poder y la paz, Ed. Sudamericana, Buenos Aires.

NYE, J. (1990). La naturaleza cambiante del poner norteamericano, GEL, Buenos Aires.

PAIVA BENEVIDES, N. G. (2011). Relações Brasil-Estados Unidos no setor de energia: do mecanismo de consultas sobre cooperação energética ao memorando de entendimento sobre biocombustíveis (20032007), Ministério das Relações Exteriores. Brasília.

ROSE, G. (1998). Neoclassical Realism and Theories of Foreign Policy en World Politics, 51 (1), October. 
RUIZ CARO, A. (2007). La seguridad energética de América Latina y el Caribe en el contexto mundial, Consejo Económico para América Latina (CEPAL), División Recursos Humanos e Infraestructura, Santiago de Chile.

SCHMIDT, B. C. (2008). The primacy of national security en S. SMITH, A. HARDFIELD, T. DUNNE, Foreign Policy: Theories, Actors, Cases, Oxford University Press, Oxford.

STRANGE, S. (1980). States and Markets, Pinter Publishers, London.

VICTOR, D. G.; YUEH, L. (2010). The new energy order en Foreign Affairs, 89 (1).

WALTZ, K. (1979). Theory of International Politics, Random House, New York.

YERGIN, D. (2006). Ensuring Energy Security en Foreign Affairs, 85 (2). 